BMJ Open

Diabetes

Research

\& Care

\section{Corneal confocal microscopy compared with quantitative sensory testing and nerve conduction for diagnosing and stratifying the severity of diabetic peripheral neuropathy}

To cite: Ferdousi $\mathrm{M}$ Kalteniece A, Azmi S, et al. Corneal confocal microscopy compared with quantitative sensory testing and nerve conduction for diagnosing and stratifying the severity of diabetic peripheral neuropathy. BMJ Open Diab Res Care 2020;8:e001801. doi:10.1136/ bmjdrc-2020-001801

Received 30 July 2020 Revised 28 October 2020 Accepted 21 November 2020

Check for updates

(C) Author(s) (or their employer(s)) 2020. Re-use permitted under CC BY-NC. No commercial re-use. See rights and permissions. Published by BMJ.

For numbered affiliations see end of article.

Correspondence to Dr Rayaz A Malik; ram2045@qatar-med.cornell. edu

\section{ABSTRACT}

Introduction Diabetic neuropathy can be diagnosed and assessed using a number of techniques including corneal confocal microscopy (CCM).

Research design and methods We have undertaken quantitative sensory testing, nerve conduction studies and CCM in 143 patients with type 1 and type 2 diabetes without neuropathy $(n=51)$, mild neuropathy $(n=47)$ and moderate to severe neuropathy $(n=45)$ and age-matched controls $(n=30)$.

Results Vibration perception threshold $(p<0.0001)$, warm perception threshold (WPT) $(p<0.001)$, sural nerve conduction velocity (SNCV) $(p<0.001)$, corneal nerve fiber density (CNFD) $(p<0.0001)$, corneal nerve branch density (CNBD) $(p<0.0001)$, corneal nerve fiber length (CNFL) $(p=0.002)$, inferior whorl length (IWL) $(p=0.0001)$ and average nerve fiber length (ANFL) $(p=0.0001)$ showed a progressive abnormality with increasing severity of diabetic neuropathy. Receiver operating characteristic curve analysis for the diagnosis of diabetic neuropathy showed comparable performance in relation to the area under the curve (AUC) but differing sensitivities and specificities for vibration perception threshold (AUC 0.79 , sensitivity $55 \%$, specificity $90 \%$ ), WPT (AUC 0.67 , sensitivity $50 \%$, specificity $76 \%$ ), cold perception threshold (AUC 0.64, sensitivity 80\%, specificity 47\%), SNCV (AUC 0.70, sensitivity 76\%, specificity 54\%), CNFD (AUC 0.71 , sensitivity $58 \%$, specificity $83 \%$ ), CNBD (AUC 0.70 , sensitivity $69 \%$, specificity $65 \%$ ), CNFL (AUC 0.68 , sensitivity $64 \%$, specificity $67 \%$ ), IWL (AUC 0.72 , sensitivity $70 \%$, specificity $65 \%$ ) and ANFL (AUC 0.72, sensitivity $71 \%$, specificity $66 \%$ ).

Conclusion This study shows that CCM identifies early and progressive corneal nerve loss at the inferior whorl and central cornea and has comparable utility with quantitative sensory testing and nerve conduction in the diagnosis of diabetic neuropathy.

\section{INTRODUCTION}

Corneal confocal microscopy (CCM) has been used to identify nerve fiber degeneration in

\section{Significance of this study}

What is already known about this subject?

- Corneal nerve loss may allow the diagnosis of diabetic neuropathy.

What are the new findings?

- There is progressive loss of corneal nerves at the central and inferior whorl region with increasing severity of diabetic neuropathy.

- Inferior whorl length was reduced while central corneal nerve fiber length was still preserved in patients without diabetic neuropathy, consistent with an early distal axonopathy.

- Corneal confocal microscopy has comparable utility with quantitative sensory testing and nerve conduction studies in the diagnosis of diabetic neuropathy.

How might these results change the focus of research or clinical practice?

- Corneal confocal microscopy may be used to diagnose and stratify the severity of diabetic neuropathy.

patients with diabetic neuropathy. ${ }^{1-5}$ It is a rapid, non-invasive nerve imaging technique with high reproducibility and moderate to high sensitivity and specificity in the diagnosis of diabetic peripheral neuropathy (DPN) ${ }^{36-10}$ To date, most studies have undertaken central corneal nerve assessment. However, recent animal and small human studies suggest that quantification of corneal nerve morphology at the inferior whorl may detect earlier nerve fiber damage. ${ }^{11-14}$ Given that DPN is a lengthdependent distal axonopathy, nerves at the inferior whorl are expected to be affected before central corneal nerves. ${ }^{15}$ Indeed, we have previously shown greater corneal nerve loss at the inferior whorl compared with the 
central cornea, ${ }^{13} 1416$ even though central corneal nerve fiber length (CNFL) is considered to be optimal for diagnosing DPN. ${ }^{17-19}$ While Pritchard et al ${ }^{13}$ reported a comparable diagnostic utility for central and inferior whorl corneal nerve length, Petropoulos et $a l^{14}$ showed that inferior whorl length (IWL) increased the sensitivity in diagnosing DPN. More recently, Kalteniece $e t a l^{16}$ combined CNFL and IWL and showed that it was more reliable than either alone, in identifying patients with diabetic neuropathy. Corneal nerve loss in larger images incorporating nerves from the center and inferior whorl has been demonstrated in patients with recently diagnosed type 2 diabetes. ${ }^{20}$ Furthermore, in our recent longitudinal study, a reduction in IWL occurred more rapidly than CNFL in patients with diabetic neuropathy. ${ }^{21}$

We have compared the utility of quantifying corneal nerve loss at the inferior whorl and central cornea with quantitative sensory testing and nerve conduction in the diagnosis and assessment of the severity of DPN.

\section{RESEARCH DESIGN AND METHODS}

\section{Study participants}

One hundred and forty-three patients with diabetes and 30 control participants underwent a comprehensive assessment of peripheral neuropathy and CCM. Each participant provided informed consent prior to participation in the study.

Patients were excluded if they had a history of connective tissue or infectious disease, malignancy, deficiency in $\mathrm{B}_{12}$ or folate, chronic renal and liver failure, current or active diabetic foot ulceration, previous ocular trauma, systemic disease other than diabetes that could cause neuropathy or affect the cornea, corneal surgery, and a history of or current contact lens wear.

\section{Clinical and peripheral neuropathy assessment}

Each participant underwent assessment of body mass index (BMI), blood pressure, glycated hemoglobin (HbA1c) and lipid profile. The simplified Neuropathy Disability Score (NDS), which assesses vibration, pinprick, temperature perception, and presence or absence of ankle reflexes, was used to stratify patients into three groups: no (NDS=0-2), mild (NDS=3-5) and moderate to severe $(\mathrm{NDS}=6-10)$ neuropathy. ${ }^{22}$

Vibration perception threshold (VPT) was assessed using a neurothesiometer (Scientific Laboratory Supplies, Wilford, Nottingham, UK), and cold perception threshold (CPT) and warm perception threshold (WPT) were tested on the dorsolateral aspect of the nondominant foot (S1) using a TSA-II NeuroSensory Analyzer (Medoc, Ramat Yishai, Israel). Electrodiagnostic studies were undertaken by a consultant neurophysiologist using a Dantec 'Keypoint' system (Dantec Dynamics, Bristol, UK) equipped with a thermistor (DISA temperature regulator, Denmark) to keep the limb temperature at $32^{\circ} \mathrm{C}-35^{\circ} \mathrm{C}$ and sural nerve conduction velocity (SNCV) was tested.

\section{Ophthalmic assessment}

Examinations of the anterior ocular segment using slit-lamp biomicroscopy and CCM examination using laser scanning CCM (Heidelberg Retina Tomograph III Rostock Cornea Module; Heidelberg Engineering, Heidelberg, Germany) were performed for both eyes according to our established protocol. ${ }^{23}$

Six images (three per eye) from the central sub-basal nerve plexus and four images (two per eye) from the inferior whorl region were selected and manually quantified using CCMetrics (The University of Manchester, Manchester, UK). Images were selected by a single expert in a masked fashion taking into account the quality, depth and variability following our previously established protocol. ${ }^{314}$ Corneal nerve fiber density (CNFD; total number of main nerves per square millimeter) (no. $/ \mathrm{mm}^{2}$ ), corneal nerve branch density (CNBD; total number of branches per square millimeter) (no. $/ \mathrm{mm}^{2}$ ), CNFL (total length of main nerves and nerve branches per square millimeter) $\left(\mathrm{mm} / \mathrm{mm}^{2}\right)$, IWL (total length of nerves per square millimeter $\left(\mathrm{mm} / \mathrm{mm}^{2}\right)$ and average nerve fiber length $(\mathrm{ANFL}=\mathrm{CNFL}+\mathrm{IWL} / 2) \quad\left(\mathrm{mm} / \mathrm{mm}^{2}\right)$ were quantified. $^{17}$

\section{Statistical analysis}

Analysis was carried out using SPSS V.22.0 for Windows. The Shapiro-Wilk test was employed to assess whether data were normally distributed. Fisher's exact test was used to test the association between two categorical variables. Analysis of variance and analysis of covariance with least significant difference correction were used for multiple comparisons between groups. All data were expressed as mean \pm SEM. $\mathrm{P}<0.05$ was considered significant. Graphs were created using GraphPad Prism (V.7.0c for Windows; GraphPad Software, La Jolla, California, USA). Receiver operating characteristic (ROC) curves were used to define the optimum cut-off points with the highest sensitivity and specificity in the diagnosis of DPN, and Youden's index (J=sensitivity+specificity-1) was measured.

\section{RESULTS}

\section{Clinical, demographic and laboratory findings}

All participant groups were matched for gender $(p=0.4)$ and ethnicity $(p=0.3)$. Age $(p=0.003)$, duration of diabetes $(\mathrm{p}=0.01)$, HbA1c $(\mathrm{p}<0.0001)$, BMI $(\mathrm{p}=0.04)$ and lowdensity lipoprotein cholesterol $(p<0.0001)$ were significantly different between all participant groups. There was no difference in high-density lipoprotein cholesterol $(\mathrm{p}=0.4)$ or triglycerides $(\mathrm{p}=0.7)$ between groups (table 1$)$.

\section{Peripheral neuropathy assessment}

VPT was significantly higher in patients with no (12.2 $\pm 1.24, \mathrm{p}=0.02)$, mild $(15.05 \pm 1.2, \mathrm{p}<0.0001)$ and moderate to severe $(25.56 \pm 1.35, \mathrm{p}<0.0001)$ neuropathy compared with controls $(7.1 \pm 1.80)$ (table 2$)$. CPT was significantly lower only in patients with mild (19.52 \pm 1.47 , 
Table 1 Demographic and clinical findings in controls and in patients with diabetes with no, mild and moderate to severe neuropathy

\begin{tabular}{|c|c|c|c|c|c|}
\hline Parameter & $\begin{array}{l}\text { Controls } \\
(n=30)\end{array}$ & $\begin{array}{l}\text { No neuropathy } \\
(n=51)\end{array}$ & $\begin{array}{l}\text { Mild neuropathy } \\
(n=47)\end{array}$ & $\begin{array}{l}\text { Moderate } \\
\text { to severe } \\
\text { neuropathy } \\
(n=45)\end{array}$ & $\begin{array}{l}\text { ANOVA } p \\
\text { value }\end{array}$ \\
\hline Gender (female/male) & $15 / 15$ & $20 / 31$ & $18 / 29$ & $16 / 29$ & 0.4 \\
\hline Ethnicity (European/non-European) & $23 / 7$ & $42 / 9$ & $31 / 16$ & $36 / 9$ & 0.3 \\
\hline $\mathrm{BMI}\left(\mathrm{kg} / \mathrm{m}^{2}\right)$ & $27.05 \pm 0.94$ & $29.09 \pm 0.86$ & $28.73 \pm 1.0$ & $31.62 \pm 1.41^{*}$ & 0.04 \\
\hline Triglycerides (mmol/L) & $1.63 \pm 0.21$ & $1.41 \pm 0.1$ & $1.53 \pm 0.14$ & $1.45 \pm 0.17$ & 0.7 \\
\hline LDL-C (mmol/L) & $2.6 \pm 0.14$ & $2.14 \pm 0.12^{*}$ & $1.9 \pm 0.15^{\star}$ & $1.7 \pm 0.08^{*} \dagger$ & $<0.0001$ \\
\hline $\mathrm{HDL}-\mathrm{C}(\mathrm{mmol} / \mathrm{L})$ & $1.67 \pm 0.1$ & $1.58 \pm 0.07$ & $1.47 \pm 0.08$ & $1.49 \pm 0.1$ & 0.4 \\
\hline
\end{tabular}

All data are expressed as mean \pm SEM.

*Significant difference compared with controls.

†Significant difference compared with no neuropathy.

ANOVA, analysis of variance; BMI, body mass index; HbA1c, glycated hemoglobin; HDL-C, high-density lipoprotein cholesterol; LDL-C, low-density lipoprotein cholesterol.

$\mathrm{p}=0.02)$ and moderate to severe $(18.99 \pm 1.55, \mathrm{p}=0.01)$ neuropathy compared with controls (25.38 \pm 2.06$)$. WPT was significantly higher in patients with no $(41.65 \pm 0.6$, $\mathrm{p}=0.01)$, mild $(43.47 \pm 0.6, \mathrm{p}<0.0001)$ and moderate to severe $(43.62 \pm 0.7, \mathrm{p}<0.0001)$ neuropathy compared with controls $(38.87 \pm 0.9)$. SNCV was significantly lower in patients with no $(43.14 \pm 1.56, \mathrm{p}=0.001)$, mild $(43.5 \pm 1.69$, $\mathrm{p}=0.002)$ and moderate to severe $(40.19 \pm 1.79, \mathrm{p}<0.0001)$ neuropathy compared with controls $(52.09 \pm 2.1)$.

\section{Corneal confocal microscopy}

CNFD was significantly lower in patients with no $(26.61 \pm 1.05, \mathrm{p}<0.0001)$, mild $(24.47 \pm 1.09, \mathrm{p}<0.0001)$ and moderate to severe $(22.4 \pm 1.14, \mathrm{p}<0.0001)$ neuropathy compared with controls $(33.71 \pm 1.3)$ (figure 1, table 2). CNBD was significantly lower in patients with no $(64.07 \pm 4.39, \mathrm{p}=0.01)$, mild $(58.49 \pm 4.76, \mathrm{p}=0.002)$ and moderate to severe $(45.60 \pm 4.50, \mathrm{p}<0.0001)$ neuropathy compared with controls $(81.52 \pm 5.54)$. CNFL was significantly lower in patients with mild $(20.84 \pm 1.004$, $\mathrm{p}=0.01)$ and moderate to severe $(19.27 \pm 1.04, \mathrm{p}=0.001)$ neuropathy compared with controls $(25.07 \pm 1.27)$. IWL was significantly lower in patients with no $(24.9 \pm 1.26$, $\mathrm{p}=0.001)$, mild $(22.28 \pm 1.31, \mathrm{p}<0.0001)$ and moderate to severe $(19.03 \pm 1.36, \mathrm{p}<0.0001)$ neuropathy compared with controls (31.69 \pm 1.66$)$. ANFL was significantly lower in patients with no $(24.1 \pm 0.98$, $\mathrm{p}=0.009)$, mild $(21.56 \pm 1.02$,

Table 2 ANCOVA with LSD correction for CCM and other measures of peripheral neuropathy in controls and in patients with diabetes with increasing severity of neuropathy

\begin{tabular}{|c|c|c|c|c|c|}
\hline Parameters & Control $(n=30)$ & $\begin{array}{l}\text { No neuropathy } \\
(n=51)\end{array}$ & $\begin{array}{l}\text { Mild neuropathy } \\
(n=47)\end{array}$ & $\begin{array}{l}\text { Moderate to severe } \\
\text { neuropathy }(n=45)\end{array}$ & $P$ value \\
\hline VPT (V) & $7.1 \pm 1.80$ & $12.2 \pm 1.24^{*}$ & $15.05 \pm 1.2^{*}$ & $25.56 \pm 1.35^{\star} \dagger \ddagger$ & $<0.0001$ \\
\hline $\mathrm{CPT}\left({ }^{\circ} \mathrm{C}\right)$ & $25.38 \pm 2.06$ & $21.68 \pm 1.44$ & $19.52 \pm 1.47^{*}$ & $18.99 \pm 1.55^{\star}$ & 0.06 \\
\hline WPT $\left({ }^{\circ} \mathrm{C}\right)$ & $38.87 \pm 0.9$ & $41.65 \pm 0.6^{*}$ & $43.47 \pm 0.6^{*} \dagger$ & $43.62 \pm 0.7^{\star} \dagger$ & $<0.001$ \\
\hline $\mathrm{SNCV}(\mathrm{m} / \mathrm{s})$ & $52.09 \pm 2.1$ & $43.14 \pm 1.56^{\star}$ & $43.5 \pm 1.69^{*}$ & $40.19 \pm 1.79^{*}$ & $<0.001$ \\
\hline CNFD (no./mm²) & $33.71 \pm 1.3$ & $26.61 \pm 1.05^{\star}$ & $24.47 \pm 1.09^{*}$ & $22.4 \pm 1.14^{*} \dagger$ & $<0.0001$ \\
\hline CNBD (no./mm²) & $81.52 \pm 5.54$ & $64.07 \pm 4.39^{\star}$ & $58.49 \pm 4.76^{*}$ & $45.60 \pm 4.5^{\star} \dagger$ & $<0.0001$ \\
\hline CNFL $\left(\mathrm{mm} / \mathrm{mm}^{2}\right)$ & $25.07 \pm 1.27$ & $23.31 \pm 0.96$ & $20.84 \pm 1.004^{*}$ & $19.27 \pm 1.04^{*} \dagger$ & 0.002 \\
\hline $\mathrm{IWL}\left(\mathrm{mm} / \mathrm{mm}^{2}\right)$ & $31.69 \pm 1.66$ & $24.9 \pm 1.26^{\star}$ & $22.28 \pm 1.31^{*}$ & $19.03 \pm 1.36^{*} \dagger$ & $<0.0001$ \\
\hline ANFL $\left(\mathrm{mm} / \mathrm{mm}^{2}\right)$ & $28.38 \pm 1.3$ & $24.1 \pm 0.98^{*}$ & $21.56 \pm 1.02^{*}$ & $19.15 \pm 1.06^{*} \dagger$ & $<0.0001$ \\
\hline
\end{tabular}

Data presented as marginal mean \pm SEM.

*Significant difference compared with controls.

†Significant difference compared with no neuropathy.

$¥$ Significant difference compared with mild neuropathy.

ANCOVA, analysis of covariance; ANFL, average nerve fiber length; CCM, corneal confocal microscopy; CNBD, corneal nerve branch density;

CNFD, corneal nerve fiber density; CNFL, corneal nerve fiber length; CPT, cold perception threshold; IWL, inferior whorl length; LSD, least significant

difference; SNCV, sural nerve conduction velocity; VPT, vibration perception threshold; WPT, warm perception threshold. 
$\mathrm{p}<0.0001)$ and moderate to severe $(19.15 \pm 1.06, \mathrm{p}<0.0001)$ neuropathy compared with controls $(28.38 \pm 1.3)$.

\section{Diagnostic utility of different measures of DPN}

The ROC curves (table 3) for quantitative sensory testing, nerve conduction and CCM for the diagnosis of diabetic neuropathy (NDS >3) (figure 2) revealed comparable area under the curve (AUC) but variable sensitivities and specificities for VPT (AUC 0.79 , sensitivity $55 \%$, specificity $90 \%$ ), WPT (AUC 0.67 , sensitivity $50 \%$, specificity $76 \%$ ), CPT (AUC 0.64, sensitivity $80 \%$, specificity $47 \%$ ), SNCV (AUC 0.70 , sensitivity $76 \%$, specificity $54 \%$ ), CNFD (AUC 0.71 , sensitivity $58 \%$, specificity $83 \%$ ), CNBD (AUC 0.70 , sensitivity $69 \%$, specificity $65 \%$ ), CNFL (AUC 0.68 , sensitivity $64 \%$, specificity $67 \%$ ), IWL (AUC 0.72 , sensitivity $70 \%$, specificity $65 \%$ ) and ANFL (AUC 0.72 , sensitivity $71 \%$, specificity $66 \%$ ).

\section{DISCUSSION}

Recommendations for the diagnosis of diabetic neuropathy require the presence of symptoms and signs and abnormal nerve conduction studies or a measure of small fiber damage if nerve conduction study (NCS) is normal. ${ }^{24}{ }^{25}$ Abnormal monofilament testing and VPT reflect large nerve fiber damage and predict an increased risk of diabetic foot ulceration, ${ }^{26}$ while thermal sensory thresholds and intraepidermal nerve fiber density identify early small nerve fiber dysfunction ${ }^{27}$ and degeneration, ${ }^{28}$ respectively. CCM is a rapid, non-invasive ophthalmic technique for the quantification of small nerve fiber damage in DPN. ${ }^{29}{ }^{30}$ Studies have shown a relationship between central corneal nerve loss and the severity of diabetic neuropathy ${ }^{20}$ 31-34 with good sensitivity and specificity for the diagnosis of DPN. ${ }^{19}{ }^{33}$ In a small early study using wide-field mapping in patients

Table 3 Diagnostic performance of quantitative sensory testing, nerve conduction and CCM in the diagnosis of diabetic peripheral neuropathy

\begin{tabular}{llllll}
\hline Neuropathy measure & AUC & P value & Sensitivity (\%) & Specificity (\%) & Youden's cut-off point \\
\hline VPT & 0.79 & $<0.0001$ & 55 & 90 & 17.37 \\
WPT & 0.67 & $<0.001$ & 50 & 76 & 44.2 \\
CPT & 0.64 & $<0.002$ & 80 & 47 & 27.3 \\
\hline SNCV & 0.70 & $<0.01$ & 76 & 54 & 45.9 \\
CNFD & 0.71 & $<0.0001$ & 58 & 83 & 23.95 \\
CNBD & 0.70 & $<0.0001$ & 69 & 65 & 58.54 \\
CNFL & 0.68 & $<0.0001$ & 64 & 67 & 21.6 \\
IWL & 0.72 & $<0.0001$ & 70 & 65 & 24.4 \\
ANFL & 0.72 & $<0.0001$ & 71 & 66 & 23.4 \\
\hline
\end{tabular}

ANFL, average nerve fiber length; AUC, area under the curve; CCM, corneal confocal microscopy; CNBD, corneal nerve branch density; CNFD, corneal nerve fiber density; CNFL, corneal nerve fiber length; CPT, cold perception threshold; IWL, inferior whorl length; SNCV, sural nerve conduction velocity; VPT, vibration perception threshold; WPT, warm perception threshold. 


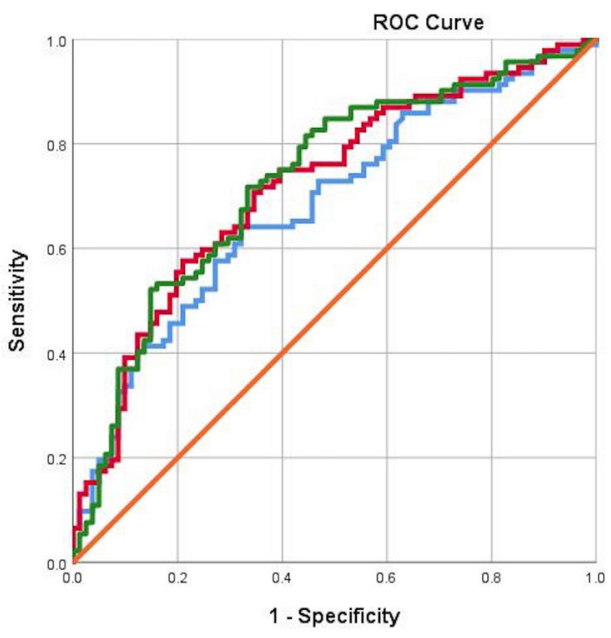

Figure 2 ROC curves for CNFL $\left(\mathrm{mm} / \mathrm{mm}^{2}\right)$, IWL $\left(\mathrm{mm} / \mathrm{mm}^{2}\right)$ and ANFL $\left(\mathrm{mm} / \mathrm{mm}^{2}\right)$. ANFL, average nerve fiber length; CNFL, corneal nerve fiber length; IWL, inferior whorl length; $\mathrm{ROC}$, receiver operating characteristic.

with diabetes with and without neuropathy, we showed greater corneal nerve loss at the inferior whorl region. ${ }^{35}$ Subsequently, in a cohort of patients with and without DPN, we demonstrated that IWL assessment enhanced the diagnostic ability of $\mathrm{CCM}^{1430}$ and it was more markedly reduced in patients with painful diabetic neuropathy. ${ }^{1636}$ More recently, in a longitudinal study in patients with DPN, we have shown more rapid corneal nerve loss at the inferior whorl compared with the central cornea. ${ }^{21}$ Furthermore, IWL rather than CNFL reduction has been associated with increased glucose variability and time above range. $^{37}$

The current study demonstrates progressive loss of corneal nerves at the central and inferior whorl region with increasing severity of DPN. ${ }^{43}$ Moreover, in patients without neuropathy we show that IWL was reduced but CNFL was still preserved, consistent with an early distal axonopathy in DPN. ${ }^{38}$ We also show progressive abnormality in the VPT, CPT, WPT and SNCV with increasing severity of DPN. In particular we show an early increase in the VPT of patients without DPN, which may reflect the early involvement of the Pacinian corpuscles. ${ }^{39}$

For the diagnosis of DPN we show comparable AUCs for quantitative sensory testing, nerve conduction and CCM. However, the sensitivity and specificity of these tests vary and may reflect the limitations of NDS in identifying early DPN. Thus VPT had a relatively low sensitivity but high specificity, while SNCV and CPT, more precise measures of large myelinated fibers, had higher sensitivity but lower specificity. However, CNFD had comparable sensitivity and specificity with VPT, while the other measures of corneal nerve fiber loss, particularly IWL and ANFL, had better sensitivity and specificity in the diagnosis of DPN. Perkins et $a \tilde{l}$ assessed 998 patients with diabetes and showed comparable AUC and sensitivity and specificity in the diagnosis of DPN using the Toronto criteria. Pritchard et $a l^{3}$ have reported $90 \%$ sensitivity and $50 \%$ specificity for CNFL compared with $80 \%$ sensitivity and
$60 \%$ specificity for IWL; however, they used fully automated software to analyze CCM images, which underestimates corneal nerve parameters compared with the manual analysis used in this study.

A limitation in the interpretation of our findings is that NDS is a relatively crude and subjective measure to diagnose and stratify the severity of DPN. Indeed, this may explain why the group designated as having no neuropathy already have abnormal VPT, CPT, SNCV and corneal nerve loss. Nevertheless, this is the largest study to date to demonstrate comparable utility of a range of CCM measures compared with quantitative sensory testing and nerve conduction studies, which are established measures of DPN.

\section{Author affiliations}

${ }^{1}$ Division of Cardiovascular Sciences, Cardiac Centre, Faculty of Biology, Medicine and Health, The University of Manchester, Manchester, UK

${ }^{2}$ Research Division, Weill Cornell Medical College in Qatar, Doha, Qatar

${ }^{3}$ Department of Experimental Medicine, University of Rome La Sapienza, Roma, Lazio, Italy

${ }^{4}$ Medicine, Weill Cornell Medical College in Qatar, Doha, Qatar

${ }^{5}$ Department of Neurology, Maastricht University, Maastricht, Limburg, The Netherlands

${ }^{6}$ Department of Clinical Neurosciences, Università di Milano - Fondazione I.R.C.C.S. Istituto Neurologico Carlo Besta, Milan, Italy

${ }^{7}$ University Department of Medicine, Central Manchester University Hospitals NHS Foundation Trust, Manchester, UK

Acknowledgements The study was supported by the Manchester Biomedical Research Centre and the Greater Manchester Comprehensive Local Research Network. Dr Mitra Tavakoli undertook corneal confocal microscopy and Dr Hassan Fadavi undertook clinical assessment and quantitative sensory testing in a proportion of patients.

Contributors MF researched data, performed the statistical analysis and wrote the manuscript. AK researched data and wrote the manuscript. SA, INP, AW, LD, SD, GP, UA and AM researched data. CGF, GL and HS reviewed and revised the manuscript. RAM designed the study and reviewed and revised the manuscript. RAM is the guarantor of this work and had full access to the data of the study and takes responsibility for the integrity of the data and the accuracy of the data analysis. The authors alone are responsible for the content and writing of the paper.

Funding The research received funding from the European Union Seventh Framework Programme FP7/2007-2013 ( ${ }^{\circ} 602273$ ), Diabetes UK (RD05/0003048), and Juvenile Diabetes Research Foundation (8-2008-362).

Competing interests None declared.

\section{Patient consent for publication Not required.}

Ethics approval The research adhered to the tenets of the Declaration of Helsinki and was approved by the NRES Committee North West - Greater Manchester East (REC reference: 14/NW/0093), North Manchester Research Ethics Committee (REC reference: 09/H1/006/38) and Central Manchester Local Research Ethics Committee (07/H1006/68).

Provenance and peer review Not commissioned; externally peer reviewed.

Data availability statement Data are available upon reasonable request. The data sets generated and analyzed during the study are available from the corresponding author on a reasonable request.

Open access This is an open access article distributed in accordance with the Creative Commons Attribution Non Commercial (CC BY-NC 4.0) license, which permits others to distribute, remix, adapt, build upon this work non-commercially, and license their derivative works on different terms, provided the original work is properly cited, appropriate credit is given, any changes made indicated, and the use is non-commercial. See: http://creativecommons.org/licenses/by-nc/4.0/.

\section{ORCID iDs}

Georgios Ponirakis http://orcid.org/0000-0002-6936-1248 
Rayaz A Malik http://orcid.org/0000-0002-7188-8903

\section{REFERENCES}

1 Alam U, Jeziorska M, Petropoulos IN, et al. Diagnostic utility of corneal confocal microscopy and intra-epidermal nerve fibre density in diabetic neuropathy. PLoS One 2017;12:e0180175.

2 Chen X, Graham J, Dabbah MA, et al. Small nerve fiber quantification in the diagnosis of diabetic sensorimotor polyneuropathy: comparing corneal confocal microscopy with intraepidermal nerve fiber density. Diabetes Care 2015;38:1138-44.

3 Kalteniece A, Ferdousi M, Adam S, et al. Corneal confocal microscopy is a rapid reproducible ophthalmic technique for quantifying corneal nerve abnormalities. PLoS One 2017;12:e0183040.

4 Petropoulos IN, Alam U, Fadavi H, et al. Corneal nerve loss detected with corneal confocal microscopy is symmetrical and related to the severity of diabetic polyneuropathy. Diabetes Care 2013;36:3646-51.

5 Perkins BA, Lovblom LE, Bril V, et al. Corneal confocal microscopy for identification of diabetic sensorimotor polyneuropathy: a pooled multinational Consortium study. Diabetologia 2018;61:1856-61.

6 Pacaud D, Romanchuk KG, Tavakoli M, et al. The reliability and reproducibility of corneal confocal microscopy in children. Invest Ophthalmol Vis Sci 2015;56:5636-40.

7 Azmi S, Ferdousi M, Petropoulos IN, et al. Corneal confocal microscopy shows an improvement in small-fiber neuropathy in subjects with type 1 diabetes on continuous subcutaneous insulin infusion compared with multiple daily injection. Diabetes Care 2015;38:e3-4.

8 Azmi S, Ferdousi M, Petropoulos IN, et al. Corneal confocal microscopy identifies small-fiber neuropathy in subjects with impaired glucose tolerance who develop type 2 diabetes. Diabetes Care 2015;38:1502-8

9 Pritchard N, Edwards K, Russell AW, et al. Corneal confocal microscopy predicts 4-year incident peripheral neuropathy in type 1 diabetes. Diabetes Care 2015;38:dc142114-5.

10 Petropoulos IN, Manzoor T, Morgan P, et al. Repeatability of in vivo corneal confocal microscopy to quantify corneal nerve morphology. Cornea 2013;32:e83-9.

11 Davidson EP, Coppey LJ, Kardon RH, et al. Differences and similarities in development of corneal nerve damage and peripheral neuropathy and in diet-induced obesity and type 2 diabetic rats. Invest Ophthalmol Vis Sci 2014;55:1222-30.

12 Cai D, Zhu M, Petroll WM, et al. The impact of type 1 diabetes mellitus on corneal epithelial nerve morphology and the corneal epithelium. Am J Pathol 2014;184:2662-70.

13 Pritchard N, Dehghani C, Edwards K, et al. Utility of assessing nerve morphology in central cornea versus Whorl area for diagnosing diabetic peripheral neuropathy. Cornea 2015;34:756-61.

14 Petropoulos IN, Ferdousi M, Marshall A, et al. The inferior Whorl for detecting diabetic peripheral neuropathy using corneal confocal microscopy. Invest Ophthalmol Vis Sci 2015;56:2498-504.

15 Müller LJ, Marfurt CF, Kruse F, et al. Corneal nerves: structure, contents and function. Exp Eye Res 2003;76:521-42.

16 Kalteniece A, Ferdousi M, Petropoulos I, et al. Greater corneal nerve loss at the inferior whorl is related to the presence of diabetic neuropathy and painful diabetic neuropathy. Sci Rep 2018;8:3283.

17 Petropoulos IN, Alam U, Fadavi H, et al. Rapid automated diagnosis of diabetic peripheral neuropathy with in vivo corneal confocal microscopy. Invest Ophthalmol Vis Sci 2014;55:2071-8.

18 Papanas N, Ziegler D. Corneal confocal microscopy: recent progress in the evaluation of diabetic neuropathy. J Diabetes Investig 2015;6:381-9.
19 Ahmed A, Bril V, Orszag A, et al. Detection of diabetic sensorimotor polyneuropathy by corneal confocal microscopy in type 1 diabetes: a concurrent validity study. Diabetes Care 2012;35:821-8.

20 Ziegler D, Papanas N, Zhivov A, et al. Early detection of nerve fiber loss by corneal confocal microscopy and skin biopsy in recently diagnosed type 2 diabetes. Diabetes 2014;63:2454-63.

21 Ferdousi M, Kalteniece A, Petropoulos I, et al. Diabetic neuropathy is characterized by progressive corneal nerve fiber loss in the central and inferior Whorl regions. Invest Ophthalmol Vis Sci 2020;61:48.

22 Young MJ, Boulton AJ, MacLeod AF, et al. A multicentre study of the prevalence of diabetic peripheral neuropathy in the United Kingdom Hospital clinic population. Diabetologia 1993;36:150-4.

23 Tavakoli M, Malik RA. Corneal confocal microscopy: a novel noninvasive technique to quantify small fibre pathology in peripheral neuropathies. J Vis Exp 2011;47. doi:10.3791/2194. [Epub ahead of print: 03 Jan 2011].

24 Tesfaye S, Boulton AJM, Dyck PJ, et al. Diabetic neuropathies: update on definitions, diagnostic criteria, estimation of severity, and treatments. Diabetes Care 2010;33:2285-93.

25 Pop-Busui R, Boulton AJM, Feldman EL, et al. Diabetic neuropathy: a position statement by the American diabetes association. Diabetes Care 2017;40:136-54

26 Abbott CA, Vileikyte L, Williamson S, et al. Multicenter study of the incidence of and predictive risk factors for diabetic neuropathic foot ulceration. Diabetes Care 1998;21:1071-5.

27 Themistocleous AC, Ramirez JD, Serra J, et al. The clinical approach to small fibre neuropathy and painful channelopathy. Pract Neurol 2014;14:368-79.

28 Javed S, Petropoulos IN, Tavakoli M, et al. Clinical and diagnostic features of small fiber damage in diabetic polyneuropathy. Handb Clin Neurol 2014;126:275-90.

29 Malik RA, Kallinikos P, Abbott CA, et al. Corneal confocal microscopy: a non-invasive surrogate of nerve fibre damage and repair in diabetic patients. Diabetologia 2003;46:683-8.

30 Petropoulos IN, Ponirakis G, Khan A, et al. Corneal confocal microscopy: ready for prime time. Clin Exp Optom 2020;103:265-77.

31 Quattrini C, Tavakoli M, Jeziorska M, et al. Surrogate markers of small fiber damage in human diabetic neuropathy. Diabetes 2007;56:2148-54.

32 Kallinikos P, Berhanu M, O'Donnell C, et al. Corneal nerve tortuosity in diabetic patients with neuropathy. Invest Ophthalmol Vis Sci 2004;45:418-22.

33 Tavakoli M, Quattrini C, Abbott C, et al. Corneal confocal microscopy: a novel noninvasive test to diagnose and stratify the severity of human diabetic neuropathy. Diabetes Care 2010;33:1792-7.

34 Edwards K, Pritchard N, Vagenas D, et al. Utility of corneal confocal microscopy for assessing mild diabetic neuropathy: baseline findings of the landmark study. Clin Exp Optom 2012;95:348-54.

35 Edwards K, Pritchard N, Gosschalk K, et al. Wide-field assessment of the human corneal subbasal nerve plexus in diabetic neuropathy using a novel mapping technique. Cornea 2012;31:1078-82.

36 Kalteniece A, Ferdousi M, Azmi S, et al. Corneal confocal microscopy detects small nerve fibre damage in patients with painful diabetic neuropathy. Sci Rep 2020;10:3371.

37 Issar T, Tummanapalli SS, Kwai NCG, et al. Associations between acute glucose control and peripheral nerve structure and function in type 1 diabetes. Diabet Med 2020;37:1553-60.

38 Malik RA, Veves A, Tesfaye S, et al. Small fibre neuropathy: role in the diagnosis of diabetic sensorimotor polyneuropathy. Diabetes Metab Res Rev 2011;27:678-84.

39 Paré M, Albrecht PJ, Noto CJ, et al. Differential hypertrophy and atrophy among all types of cutaneous innervation in the glabrous skin of the monkey hand during aging and naturally occurring type 2 diabetes. J Comp Neurol 2007;501:543-67. 